\title{
Viral organization of human proteins
}

\author{
Stefan Wuchty
}

\section{Corresponding author:}

\section{Stefan Wuchty}

Northwestern Institute of Complexity

Northwestern University

600 Foster Street, Evanston, IL 60201

Present address:

National Cancer Institute

National Institutes of Health

37 Convent Drive, Bethesda, MD 20892

Voice: +1 3014432787

E-Mail: wuchtys@mail.nih.gov 


\begin{abstract}
A compilation of experimentally verified interactions between HIV-1 and human proteins allows insights into the intricate interplay between viral and host proteins on a large scale. We find that HIV-1 predominantly targets rich-clubs, human proteins that are not only well connected but also strongly intertwined among each other. These assemblies of proteins putatively serve as an infection gateway, allowing the virus to take control of the human host by reaching protein pathways and diversified cellular functions in a pronounced and focused way. In particular, HIV-1 utilizes its small number of proteins in a combinatorial manner, exerting a significant influence on pathways that deal with transcriptional, translational and degradation processes. Surprisingly, the small repertoire of HIV proteins also interferes loosely with many signaling and regulation pathways, suggesting that a widespread involvement in such pathways secures the control of the host cell. Such insights offer novel perspectives to investigate the progression of HIV infection and potentially can contribute to our abilities to fight this virus.
\end{abstract}


The determination of webs of protein interactions ${ }^{1,2,3,4}$ and protein complexes $5,6,7,8,9$ in many different single and multi-cellular organisms progresses at a fast pace, peaking in attempts to determine the human interactome in various ways $10,11,12,13,14$. Although such webs of intracellular interactions are increasingly well characterized, little is known about large-scale maps of protein interactions between cells. As such, investigating host-pathogen interactions is a crucial step toward a thorough understanding of an organisms pathogenesis, providing an essential foundation for the development of effective therapeutic and prevention strategies to combat diseases. Uetz et al. released the first small map of computationally inferred physical protein interactions between the human host and the Kaposi-Sarcoma associated Herpesvirus (KSHV) as well as the Varicella-Zoster-Virus (VZV) ${ }^{15}$. In a different approach Calderwood et al. ${ }^{16}$ experimentally determined a map of physical protein interactions between the Epstein-Barr-Virus and the human host. Recently, Bandyopadhyay et al. ${ }^{17}$, identified subnetworks of virus-host proteins that are expressed at different stages of the HIV-infection.

Rich-clubs of proteins as viral targets. Here, we utilize a compilation of 1, 199 experimentally verified physical and regulatory interactions between 15 HIV-1 and 904 human proteins that are embedded in a web of 12, 542 connections between 66, 438 human proteins ${ }^{14,18,19,20,21}$. Among those we identify 341 interactions, representing activating or up-regulating activities of HIV on human proteins ('positive interactions'), while the data set provides 220 interactions that reflect the virus inhibiting activities ('negative interactions').

Considering a graphical depiction of the web of all host-viral interactions in Fig. 1a, we not only see an uneven distribution of the number of interactions viral proteins are involved in but also observe many host proteins that are targeted by 
more than one viral protein. As such, we will show topological features of the human web that are potential targets of HIV-1 as well as investigate combinatorial patterns virus employ to take control of the host cell. In contrast to other protein interaction networks of eukaryotic organisms, such as S. cerevisiae, C. elegans and D. melanogaster ${ }^{22,23}$ the human interactome is composed of an oligarchy of highly interacting and intertwined nodes. Such a rich-club phenomenon $^{22}$ is quantified by the fraction of edges among nodes that have at least a certain number of neighbors $k$ in the actual and randomized networks. As such, the rich-club coefficient $\rho(k)$ points to the presence of a core of highly intertwined nodes with degree of at least $k$ if $\rho(k)>1$. In the absence of this phenomenon (i.e. $\rho(k)<1$ ) networks are dominated by many well defined functional communities which are sparsely connected by highly interacting proteins ${ }^{22,24}$. In the human interactome we find a strong rich-club signal (i.e. $\rho>1$ ) among proteins with increasing degree in the human interactome (Fig. 1b). As indicated the absence of any rich-clubs would point to the presence of loosely connected sub-networks. Therefore, such a network would break into many disconnected parts ${ }^{23}$. In contrast, networks that show rich-club behavior always keep their integrity by maintaining one connected component. As already indicated in Fig. 1a, we find one connected component that covers all viral and human proteins. If the placement of host-pathogen interactions was a random process, such a network would break into numerous disconnected pieces. We randomly choose sets of human proteins that are targeted by HIV-1 proteins, allowing us to find that the presence of a connected component is statistically significant $\left(P<10^{-3}\right)$. In the same way, we find that the three connected components in bipartite networks of positive and negative interactions are of the same significance as well. The features of rich-clubs in the underlying human interactome are potentially proteomic targets that a pathogen might utilize. 
Indeed, we find an enrichment of proteins in rich-clubs that are targeted by HIV-1. In particular, we find a recurrence of this pattern if we focus on the subsets of negative and positive interactions (Fig. 1c). As indicated on a qualitative basis, the number of human host proteins a viral protein targets is not evenly distributed (Fig. 1a). In fact, we find that Tat, Nef and Vpr - viral proteins that predominantly interfere with regulatory host processes - work on far more human proteins than a simple average would suggest (see SOMTable 1). In Fig. 1d, we observe that one viral protein acts on the majority of human proteins, while a minority is targeted by up to 6 viral proteins, a pattern we also find for positive and negative interactions. Such distributions roughly decay exponentially, an inhomogeneity that is visually captured in the graphical representation of virus-host interactions in Fig. 1a. Assuming that the total number of HIV-1 proteins reflects the importance and centrality of a targeted host protein, we expect that the mean number of viral proteins increases in rich-clubs. Indeed, we find such an increasing trend for the total set of host-virus interactions as well as positive interactions in Fig. 1d. Although we observe that negative interactions lack such a correlation, our results suggest that samples of highly connected and intertwined proteins provide topological features the virus utilizes as a gateway to take over control over the host cell.

The role of protein pathways as viral targets. Another level of proteomic organization are protein pathways. As a reliable source, we obtained 913 manually curated pathways from the Protein Interaction Database (PID). As a result, we not only find that human protein proteins strongly vary in the number of involved proteins (inset, SOMFig. 1b) but also share proteins to a large extent (SOMFig. 1a). Emphasizing the role of protein hubs to be involved in numerous protein pathways (SOMFig. 1b), we anticipate that the pathogen also takes advantage of this host feature. Indeed, we find that proteins that appear in 
rich-clubs of increasing degree appear in an increasing number of pathways (Fig. $2 \mathrm{a}$ ), a result that also appears in the smaller subset of positive and negative interactions (SOMFig. 2a). A corollary of this hypothesis suggests that the comparably small number of human proteins that HIV-1 targets would allow the virus to reach a larger number of pathways than by chance alone. Although a justified argument, we find quite the opposite in the inset of Fig. 2a. In fact, the random selection of targeted human proteins reaches a larger total number of protein pathways $\left(P<10^{-3}\right)$ than in the actual case. Analogously, we find the same results for human proteins that are either positively or negatively influenced by the virus (SOMFig. 2b), indicating the relevance of a focused placement of viral links in the human host pathways that allow to take over control of the host cell to the largest extent. On a different note, we hypothesize that the predominance of targeting hubs as potential mediators of a viral infection also has an aspect of variety. Assuming that the virus tries to optimize its pathway 'reach', we expect that HIV-1 might target proteins, that are involved in many different pathways. As a measure of diversity, we define the pathway participation coefficient: if a protein predominantly interacts with partners that are members of the same pathway, this measure tends toward 1 , while the opposite holds if the interaction partners of the protein in question are distributed among many different pathways. Accounting for all human proteins, we largely observe that interactions of a single protein occur in a variety of pathways, while relatively few interactions are confined to a small number of pathways (Fig. 2b). Focusing on the subsets of targeted human proteins, we observe a reinforcement of the initial diversity signal. Supporting our initial hypothesis, this result indicates that the virus aims to reach into as many pathways as possible by diversifying the reach of host-pathogen interactions to the largest extent.

Combinatorial utilization of viral proteins. As mentioned previously, the 
observation that a bipartite network of interactions between HIV-1 and human proteins is one connected component strongly suggests that combinations of viral proteins target the same subsets of human proteins. In Fig. 3a, we construct a network, linking viral proteins if they share a subset of human proteins they target together. In particular, we weight each link by the size of the shared subset of targeted human proteins. We observe that Tat dominates such a network, largely working the same targets predominantly in combination with $\mathrm{Vpr}$ and Nef as well as Vif, while there also exists a weaker link between Vpr and Nef. The richness of the underlying data also allows us to elucidate such combinations of regulatory links between viral and human proteins that share similar functions. In Fig. 3b, we observe that Tat down-regulates subunits of the $26 \mathrm{~S}$ particle of the human proteasome in combination with Vif. This observation largely indicates the virus objective to mute the apparatus which removes disposed proteins, activities that potentially impair activities to produce new viral protein particles. In the same way, HIV-1 interferes with immunological responses upon an infection. As such, human proteins that participate in immunological pathways such as interleukines and major histocompatibility complexes (MHC) are largely controlled by a combination of viral proteins. Interleukines - proteins that play a pivotal role in activating signal pathways for the stimulation of B and NK cells ${ }^{25}$ - are largely controlled by Nef, Tat and Vpr (Fig. 3c). A different class of proteins involved in defensive activities, MHC molecules, play a vital role in the complex immunological dialogue. All MHC molecules receive polypeptides from inside the cells and display them on the cells exterior surface for recognition by T cells. An interaction with the T-cells triggers an immune response if polypeptides of foreign origin are identified ${ }^{26}$. As such, one of the objectives of the virus is to silence such pathways, preventing the release of signals of a cellular disturbance, as exemplified by a strong inhibiting and 
binding activity on MHC subunits by Nef and Tat. Similarly, classes of interferones can trigger an immune response to a RNA virus infection by their secretion when abnormally large amounts of dsRNA are found in a cell, leading to the attenuation of the cells ability to initiate translation. This induced shortage of protein production not only interferes with viral replication, but also inhibits normal cell ribosome function, killing both the virus and the host cell.

Furthermore, interferones lead to the up-regulation of MHC I and therefore to an increased presentation of viral peptides to cytotoxic CD8 T cells, as well as to a change in the proteasome which leads to increased production of MHC I compatible peptides. While MHC I subunits are predominantly blocked by Tat and Nef, IFN- $\gamma$ is controlled by capsid, matrix, Nef, Tat and Vpr (data not shown). Interferones can also cause increased p53 ${ }^{27}$ activity in virus infected cells, acting as inducers, promoting apoptosis and limiting the ability of the virus to spread ${ }^{28,29}$. Indeed, we find that Tat in putative combination with Nef, Vpr and reverse transcriptase inhibits p53 and other tumor suppressor genes and oncogenes (Fig. 3d), that play important roles in other signaling pathways. In particular, we observe that NF- $\kappa \mathrm{B}$ is largely activated by this combination of viral proteins, indicating a particular role of the NF- $\kappa$ B pathway as a focus of virus interference and tumor emergence. Recently, reports indicated that this pathway does not only play a primary role in many different types of cancer, but can easily be activated by low mutational activity of the underlying pathway genes ${ }^{30,31}$. Similarly, we observe that especially combinations of Tat, Nef and Vpr predominantly activate an array of protein kinases. Among others, we find a prominent protagonists such as phoshatidylinositol-3-kinase (PI3K) where mutations in the latter protein are frequent in many breast cancers ${ }^{32,33,34}$, implicating that both the NF- $\kappa \mathrm{B}$ and PI3K pathway are pivotal not only for tumor emergence but also for the invasion process of HIV-1. 
Entanglement of pathways. While we focused on single proteins in the previous section, we take the next step by determining a network of pathways if they share proteins that interact with the virus. Utilizing pathways from the Pathway Interaction Database we assess the significance of weighted links between attacked protein pathways by a two-tailed Fisher's test ${ }^{35}$. Applying a threshold of $P<10^{-3}$ we find that the resulting web largely breaks into four functionally distinct parts (Fig. 4). In particular, densely connected components refer to pathways that play a role in transcription and mRNA processing activities as well as degradation and translational activities. As indicated in Fig. 3, HIV-1 utilizes its proteomic material in a combinatorial way to interact with proteins that are involved in such functions and to secure the production of further virus particles. Although we found hints that HIV-1 also interacts with a few interesting signaling proteins (Fig. 3d), we surprisingly find a large and loosely connected component in the pathway network, composed of numerous prominent signaling and regulation pathways, clearly suggesting that the virus needs to interact with a considerable number of such pathways to seize control of the cell. As indicated before, we also find that positive ties largely govern transcription and mRNA processing pathways, while negative interaction influence entirely degradation pathways (data not shown). In SOMTable 2, we list all these pathways we show in Fig. 4. In particular, we find a pathway that deals with signaling events involving hepatocyte growth factor receptor c-met (\#85) to be a major hub. In particular, the proteins which are targeted by the virus are largely prominent signaling proteins such as MAP kinases and PI3K, which also appear in many other signaling and regulation pathways. As such, these observations suggest that the proteins that are in the intersection of many different pathways are key players for understanding the ways HIV gains control of the human host.

Discussion. HIV-1 invokes intricate processes with a remarkably low number of 
proteins to take control of the human host cell. In particular, the subtle structure of the human interactome reveals sites that are not only topologically important, but also are significantly targeted by the HIV-virus. Compensating for its low number of proteins, combinations of pathogen proteins allow the virus to simultaneously act on many different human proteins. Such subsets reach into functional entities assuring largest diversity in a focused way. Remarkably, such combinations of virus proteins interfere with a variety of different pathways in order to take control of the human host, ultimately utilizing it for the virus own survival. Therefore, untangling the intricate web of intertwined pathways is of utmost importance for a thorough understanding of the virus pathogenesis. In the light of these observations proteins that are shared by a large number of relevant pathways might be key players to understand the subtle molecular strategies a virus employs to take over control of a host cell. In addition, the analysis of the entanglement of signaling and regulation pathways may uncover molecular and functional Achilles heels that might help to hamper the virus in a systematic way

36. Therefore, a web of well defined host-pathogen interactions offers the opportunity to consider viral systems as naturally perturbed biological systems that can be utilized to identify and disentangle relevant pathways in different cellular contexts, ultimately allowing us to eradicate other pathogen driven diseases that plague human kind.

\section{Materials and Methods}

\section{Human HIV Protein Interactions}

We utilize a compilation of 1, 199 experimentally obtained protein interactions between the human host and HIV-1, accounting for interactions that have been found in vital cells in the human immune system such as helper T cells, 
macrophages and dendritic cells

(http://www.ncbi.nlm.nih.gov/RefSeq/HIVInteractions/). 341 interactions

describe activation and/or up-regulating activities of HIV on human proteins

('positive' interactions), and 220 interactions depict inhibiting activities of the virus ('negative' interactions).

\section{Human Protein Interactions and Pathways}

Collecting pairwise protein interactions in $H$. sapiens from public databases ${ }^{18,20}$ and experimental results $11,14,19,21$, we obtain a network of 12,542 proteins embedded in 66, 438 physical interactions. As a source of reliable human protein pathway information we utilized 913 annotated pathways from the Pathway Interaction Database (http://pid.nci.nih.gov).

\section{Rich-Club Coefficient}

The so-called rich-club phenomenon is quantitatively defined by the rich-club coefficient $\Phi(k)^{22}$. Denoting by $E_{\geq k}$ the number of edges among the $N_{\geq k}$ nodes which have at least $k$ interaction partners, the rich-club coefficient is expressed as

$$
\Phi(k)=\frac{2 E_{\geq k}}{N_{\geq k}\left(N_{\geq k}-1\right)},
$$

where $N_{\geq k}\left(N_{\geq k}-1\right) / 2$ represents the maximally possible number of edges among the $N_{\geq k}$ nodes. An appropriate choice for normalizing the rich-club coefficient is provided by the ratio

$$
\rho(k)=\frac{\Phi(k)}{\Phi_{r}(k)},
$$

where $\Phi_{r}(k)$ is the rich-club coefficient of a random network with the same degree distribution $P(k)$. The choice of pairs of links, whose end nodes are exchanged, allows us to obtain such a randomized network where the degree 
distribution is preserved ${ }^{22,37}$. In order to have a reasonably large ensemble, we repeat the randomization process 10,000 times. Binning nodes according to their degrees $k$ we obtain a degree dependent mean value of the rich-club coefficient by averaging over all $\rho$ 's in each bin. A ratio larger than one, $\rho>1$, is the actual evidence for the presence of a rich-club phenomenon, an increase in the interconnectivity of large degree nodes compared to the random case. This process is well displayed by the presence of an oligarchy of highly interacting nodes that are well connected among each other. A ratio $\rho(k)<1$ points to a lack of interconnectivity among large degree nodes which are separated in distinguishable modules.

\section{Enrichment}

Each rich club where each protein has at least $k$ interactions $N_{\geq k}$ is represented as a subset of all proteins $N$ in the underlying network, $N_{\geq k} \in N$. In order to obtain an estimate if proteins targeted by the virus are overrepresented in a rich-club, we calculate the corresponding fraction of attacked proteins $f_{a, \geq k}=\left|N_{a, \geq k}\right| /\left|N_{\geq k}\right|$ in the underlying rich club $N_{\geq k}$. As a null hypothesis, we assume that the virus targets human proteins randomly. Determining the randomized fraction of targeted proteins $f_{r, a, \geq k}$, we define $E_{a, \geq k}=f_{a, \geq k} / f_{r, a, \geq k}$ as the enrichment of attacked proteins in a rich club. Averaging $E$ over 10, 000 randomizations rich clubs are enriched with attacked proteins if $E>1$ and vice versa. 


\section{Pathway Participation Coefficient}

For each protein that is part of at least one pathway, we define the pathway participation coefficient of a protein $i$, as

$$
P_{i}=\sum_{s=1}^{N}\left(\frac{n_{i s}}{\sum_{s=1}^{N} n_{i s}}\right)^{2},
$$

where $n_{i s}$ is the number of links protein $i$ has to proteins in pathway $s$ out of $N$ total pathways. If a protein predominantly interacts with partners that are members of the same pathway, we find that $P$ tends to 1 , while the opposite holds if the interaction partners are distributed among many different pathways.

\section{Significance of Links between attacked Pathways}

Determining the significance of links between pathways if they share proteins that interact with viral proteins, we form a $2 \times 2$ contingency table for each pair of pathways. In particular, we define $\alpha$ as the number of shared proteins under attack by the virus while $\beta$ is the number of remaining proteins in a pair of overlapping pathways. Analogously, we define $\gamma$ as the number of attacked proteins and $\delta$ as the number of remaining proteins in all the other pathways ${ }^{35}$. The probability of obtaining any such set of values randomly is given by the hypergeometric distribution:

$$
p^{*}=\frac{\left(\begin{array}{c}
\alpha+\beta \\
\alpha
\end{array}\right)\left(\begin{array}{c}
\gamma+\delta \\
\gamma
\end{array}\right)}{\left(\begin{array}{c}
N \\
\alpha+\gamma
\end{array}\right)},
$$

where $N=\alpha+\beta+\gamma+\delta$. In order to investigate the two tails of the underlying distribution we construct all possible contingency tables by keeping the sum of rows and columns constant. The $P$-value to reject the null hypothesis being the independence of rows and olumns in the contingency table is the sum of the probabilities $p_{i}$, of all contingency tables $i$ where $p_{i} \leq p^{*}$. 


\section{References}

[1] Reguly, T., Breitkreutz, A., Boucher, L., Breitkreutz, B., Hon, G., Myers, C., Parsons, A., Friesen, H., Oughtred, R., Tong, A., Stark, C., Ho, Y., Botstein, D., Andrews, B., Boone, C., Troyanskya, O., Ideker, T., Dolinski, K., Batada, N., and Tyers, M. Comprehensive curation and analysis of global interaction networks in saccharomyces cerevisiae. J. Biol. 5(11) (2006).

[2] Giot, L., Bader, J., Brouwer, C., Chaudhuri, A., Kuang, B., Li, Y., Hao, Y., Ooi, C., Godwin, B., Vitols, E., Vijayadamodar, G., Pochart, P., Machineni, H., Welsh, M., Kong, Y., Zerhusen, B., Malcolm, R., Varrone, Z., Collis, A., Minto, M., Burgess, S., McDaniel, L., Stimpson, E., Spriggs, F., Williams, J., Neurath, K., Ioime, N., Agee, M., Voss, E., Furtak, K., Renzulli, R., Aanensen, N., Carrolla, S., Bickelhaupt, E., Lazovatsky, Y., DaSilva, A., Zhong, J., Stanyon, C., Finley Jr., R., White, K., Braverman, M., Jarvie, T., Gold, S., Leach, M., Knight, J., Shimkets, R., McKenna, M., Chant, J., , and Rothberg, J. A protein interaction map of drosophila melanogaster. Science 302, 1727-1736 (2004).

[3] Li, S., Armstrong, C., Bertin, N., Ge, H., Milstein, S., Boxem, M., Vidalain, P., Han, J., Chesneau, A., Hao, T., Goldberg, D., Li, N., Martinez, M., Rual, J., Lamesch, P., Xu, L., Tewari, M., Wong, S., Zhang, L., Berriz, G., Jacotot, L., Vaglio, P., Reboul, J., Hirozane-Kishikawa, T., Li, Q., Gabel, H., Elewa, A., Baumgartner, B., Rose, D., Yu, H., Bosak, S., Sequerra, R., Fraser, A., Mango, S., Saxton, W., Strome, S., Heuvel, S. V. D., Piano, F., Vandenhaute, J., Sardet, C., Gerstein, M., Doucette-Stamm, L., Gunsalus, K., Harper, J., Cusick, M., Roth, F., Hill, D., and Vidal, M. A map of the 
interactome network of the metazoan c. elegans. Science 303, 540-543 (2004).

[4] Rain, J.-C., Selig, L., DeReuse, H., Battaglia, V., Reverdy, C., Simon, S., Lenzen, G., Petel, F., Wojcik, J., Schächter, V., Chemama, Y., Labigne, A., and Legrain, P. The protein-protein interaction map of Helicobacter pylori. Nature 409, 211-215 (2001).

[5] Krogan, N., Cagney, G., Yu, H., Zhong, G., Guo, X., Ignatchenko, A., Li, J., Pu, S., Datta, N., and et al., A. T. Global landscape of protein complexes in the yeast saccharomyces cerevisiae. Nature 440, 637-643 (2006).

[6] Gavin, A., Bösche, M., Krause, R., Grandi, P., Marzioch, M., Bauer, A., Schultz, J., Rick, J., Michon, A.-M., Cruciat, C.-M., Remor, M., Böfert, C., Schelder, M., Brajenovic, M., Ruffner, H., Merino, A., Klein, K., Hudak, M., Dickson, D., Rudi, T., Gnau, V., Bauch, A., Bastuck, S., Huhse, B., Leutwein, C., Heurtier, M.-A., Copley, R., Edelmann, A., Querfurth, E., Rybin, V., Drewes, G., Raida, M., Bouwmeester, T., Bork, P., Seraphin, B., Kuster, B., Neubauer, G., and Superti-Furga, G. Functional organization of the yeast proteome by systematic analysis of protein complexes. Nature 415, 141-147 (2002).

[7] Gavin, A., Aloy, P., Grandi, P., Krause, R., Marzioch, M. B., Rau, C., Jensen, L., Bastuck, S., Dümpelfeld, B., Edelmann, A., Heurtier, M.-A., Hoffman, V., Höfert, C., Klein, K., Hudak, M., Michon, A.-M., Schelder, M., Schirle, M., Remor, M., Rudi, T., Hooper, S., Bauer, A., Bouwmeester, T., Casari, G., Drewes, G., Neubauer, G., Rick, J., Kuster, B., Bork, P., Russell, R., and Superti-Furga, G. Proteome survey reveals modularity of the yeast cell machinery. Nature 440, 631-636 (2006). 
[8] Ho, Y., Gruhler, A., Heilbut, A., Bader, G., Moore, L., Adams, S.-L., Millar, A., Taylor, P., Bennett, K., Boutillier, K., and coauthors, . Systematic identification of protein complexes in saccharomyces cerevisiae by mass spectrometry. Nature 415, 180 - 183 (2002).

[9] Sprinzak, E., Altuvia, Y., and Margalit, H. Characterization and prediction of protein-protein interactions within and between complexes. Proc. Natl. Acad. Sci. USA 104, 14718-14723 (2006).

[10] Lehner, B. and Sanderson, C. A protein interaction framework for human mrna degradation. Genome Res. 14, 1315-1323 (2004).

[11] Ramani, A., Bunescu, R., Mooney, R., and Marcotte, E. Consolidating the set of known human protein-protein interactions in preparation for large-scale mapping of the human interactome. Genome Biol. 6(5), R40 (2005).

[12] Gandhi, T., Zhong, J., Mathivanan, S., Karthick, L., Chandrika, K., Mohan, S., Sharma, S., Pinkert, S., Nagaraju, S., Periaswamy, B., Mishra, G., Nandakumar, K., Shen, B., Deshpande, N., Nayak, R., Sarker, M., Boeke, J., Parmigiani, G., Schultz, J., Bader, J., and Pandey, A. Analysis of the human protein interactome and comparison with yeast, worm and fly interaction datasets. Nat. Genetics 38(3), 285-293 (2006).

[13] Rhodes, D., Tomlins, S., Varambally, S., Mahavisno, V., Barrette, T., Kalyana-Sundaram, S., Ghosh, D., Pandey, A., and Chinnaiyan, A. Probabilistic model of the human protein-protein interaction network. Nat. Biotech. 23(8), 951-959 (2005).

[14] Stelzl, U., Worm, U., Lalowski, M., Haenig, C., Brembeck, F., Goehler, H., Stroedicke, M., Zenkner, M., Schoenherr, A., Koeppen, S., Timm, J., 
Mintzlaff, S., Abraham, C., Bock, N., Klietzmann, S., Goedde, A., Toksoez, E., Droege, A., Krobitsch, S., Korn, B., Birchmeier, W., Lehrach, H., and Wanker, E. A human protein-protein interaction network: A resurce for annotationg the proteome. Cell 122, 957-968 (2005).

[15] Uetz, P., Dong, Y.-A., Zeretzke, C., Atzler, C., Baiker, A., Berger, B., Rajagopala, S., Roupelieva, M., Rose, D., Fossum, E., and Haas, J. Herpesviral protein networks and their interaction with the human proteome. Science 311, 239-242 (2006).

[16] Calderwood, M., Venkatesan, K., Xing, L., Chase, M., Vazquez, A., Holthaus, A., Ewnce, A., Li, N., and et al. Epstein-barr virus and virus human protein interaction maps. Proc. Natl. Acad. Sci. USA 104, 7606-7611 (2007).

[17] Bandyopadhyay, S., kelley, R., and Ideker, T. Discovering regulated networks during hiv-1 latency and reactivation. In Proceedings of the Pac. Symp. Biocomput., 354-366, (2006).

[18] Mishra, G., Suresh, M., Kumaran, K., Kannabiran, N., Suresh, S., Bala, P., Shivkumar, K., Anuradha, N., Reddy, R., Raghavan, T., Menon, S., Hanumanthu, G., Gupta, M., Upendran, S., Gupta, S., Mahesh, M., Jacob, B., Matthew, P., Chatterjee, P., Arun, K., Sharma, S., Chandrika, K., Deshpande, N., Palvankar, K., Raghavnath, R., Krishnakanth, K., Karathia, H., Rekha, B., Rashmi, N., Vishnupriya, G., Kumar, H., Nagini, M., Kumar, G., Jose, R., Deepthi, P., Mohan, S., Gandhi, T., Harsha, H., Deshpande, K., Sarker, M., Prasad, T., and Pandey, A. Human protein reference database 2006 update. Nucl. Acids Res. 34, D411-D414 (2006). 
[19] Rual, J., Venkatesan, K., Hao, T., Hirozane-Kishikawa, T., Dricot, A., Li, N., Berriz, G., Gibbons, F., Dreze, M., Ayivi-Guedehoussou, N., Klitgord, N., Simon, C., Boxem, M., Milstein, S., Rosenberg, J., Goldberg, D., Zhang, L., Wong, S., Franklin, G., Li, S., Albala, J., Lim, J., Fraughton, C., Llamosas, E., Sebiha-Cevik, S., Bex, C., Lamesch, P., Sikorski, R., Vandenhaute, J., Zoghbi, H., Smolyar, A., Bosak, S., Sequerra, R., L. Doucette-Stamm, M. C., Hill, D., Roth, F., and Vidal, M. Towards a proteome-scale map of the human protein-protein interaction network. Nature 437, 1173-1178 (2005).

[20] Joshi-Tope, G., Gillespie, M., Vastrik, I., D’Eustachio, P., Schmidt, E., de Bono, B., Jassal, B., Gopinath, G., Wu, G., Matthews, L., Lewis, S., Birney, E., and Stein, L. Reactome: a knowledgebase of biological pathways. Nucl. Acids Res. 33, D428-D432 (2005).

[21] Ewing, R., Chu, P., Elisma, F., Li, H., Taylor, P., Climie, S., McBroom-Cerajewski, L., Robinson, M., O’Connor, L., Li, M., Taylor, R., Dharsee, M., Ho, Y., Heilbut, A., Moore, L., Zhang, S., Ornatsky, O., Bukhman, Y., Ethier, M., Sheng, Y., Vasilescu, J., Lambert, M. A.-F. J.-P., Duewel, H., Stewart, I., Kuehl, B., Hogue, K., Colwill, K., Gladwish, K., Muskat, B., Kinach, R., Adams, S.-L., Moran, M., Morin, G., Topaloglou, T., and Figeys, D. Large-scale mapping of human protein protein interactions by mass spectrometry. Mol. Syst. Biol. 3(89) (2007).

[22] Colizza, V., Flamini, A., Serano, M., and Vespignani, A. Detecting rich-club ordering in complex networks. Nat. Physics 2, 110-115 (2006).

[23] Wuchty, S. Evolutionary conservation of motif constituents within the yeast protein interaction network. PloS One 2(3), e335 (2007). 
[24] Han, J., Bertin, N., Hao, T., D.S.Goldberg, Berriz, G., Zhang, L., Dupuy, D., Walhout, A., Cusick, M., Roth, F., and Vidal, M. Evidence for dynamically organized modularity in the yeast protein-protein interaction network. Nature 430, 88-93 (2004).

[25] Waldmann, T. The biology of interleukin-2 and interleukin-15: implications for cancer therapy and vaccine design. Nat. Rev. Immun. 6, 595-601 (2006).

[26] Alberts, B., Bray, D., Lewis, J., Raff, M., Roberts, K., and Watson, J. Molecular Biology of the Cell. Garland Publishing, Inc., New York, (1994).

[27] Vogelstein, B., Lane, D., and Levine, A. Surfing the p53 network. Nature 408, 307-310 (2000).

[28] Moiseeva, O., Mallette, F., Mukhopadhyay, U., Moores, A., and Ferbeyre, G. Dna damage signaling and p53-dependent senescence after prolonged beta-interferon stimulation. Mol. Biol. Cell 17(4), 1583-1592 (2006).

[29] Takaoka, A., Hayakawa, S., Yanai, H., D.Stoiber, Negishi, H., Kikuchi, H., Sasaki, S., Imai, K., Shibue, T., Honda, K., and Taniguchi, T. Integration of interferon-a/b signalling to p53 responses in tumour suppression and antiviral defence. Nature 424, 516-523 (2003).

[30] Keats, J., Fonseca, R., Chesi, M., Schop, R., Baker, A., Chng, W.-J., Wier, S., Tiedemann, R., and et al. Promiscuous mutations activate the noncanonical nf- $\kappa \mathrm{b}$ pathway in multiple myeloma. Cancer Cell 12, 131-144 (2007).

[31] Annunziata, C., davis, R., Demchenko, Y., Bellamy, W., Gabrea, A., Zhan, F., lenz, G., Hanamura, I., Wright, G., and et al. Frequent engagement of the classical and alternative $\mathrm{nf}-\kappa \mathrm{b}$ pathways by diverse genetic abnormalities in multiple myeloma. Cancer Cell 12, 115-130 (2007). 
[32] Samuels, Y., Wang, Z., Bardelli, A., Silliman, N., Ptak, J., Szabo, S., Yan, H., Gazdar, A., and et al. High frequeny of mutations of the pik3ca gene in human cancers. Science 304, 554 (2004).

[33] Lee, J., Soung, Y., Kim, S., Lee, H., Park, W., Nam, S., Kim, S., Lee, J., Yoo, N., and Lee, S. Pik3ca gene is frequently mutated in breast carcinomas and hepatocellular carcinomas. Oncogene 24, 1477-1480 (2005).

[34] Wood, L., Parsons, D., Jones, S., Lin, J., Sjöblom, T., Leary, R., Shen, D., Boca, S., Barber, T., Ptak, J., and et al. The genomic landscapes of human breast and colorectal cancers. Science 318, 1108-1113 (2007).

[35] Francesconi, M., Remondini, D., Neretti, N., Sedivy, J., Cooper, L., Verondini, E., Melanesi, L., and Castellani, G. Reconstructing networks of pathways via significance analysis of their intersections. $B M C$ Bioinformatics 9(Suppl 4), S9 (2008).

[36] Tan, S.-L., Ganji, G., Paeper, B., Proll, S., and Katze, M. Systems biology and the host response to viral infection. Nature Biotechn. 25(12), 1383 1389 (2007).

[37] Maslov, S. and Sneppen, K. Specificity and stability in topology of protein networks. Science 296, 910-913 (2002). 
Figure 1: (a) Graphical representation of the network of all protein interactions between HIV-1 and human proteins. (b) The distribution of the mean rich-club coefficient $\rho$ significantly increases with higher numbers of interaction partners in the human protein interaction network, suggesting the presence of an oligarchy of highly interacting and intertwined proteins. (c) Determining the enrichment of all human proteins that are targeted by HIV-1, we find that especially highly connected proteins are predominantly affected by the virus. Considering only human host proteins that are either positively or negatively influenced by the virus we observe similar trends. (d) As another biologically significant characteristic of the viral infection, we observe that host proteins can be targeted by more than one viral protein, results that are consistent with the smaller subsets of positive and negative interactions. Calculating the mean number of targeting viral proteins in rich-clubs, we obtain increasing numbers toward higher connected, while we find almost no correlation for host proteins that are negatively influenced by the virus (inset).

Figure 2: (a) Another level of proteomic organization are protein pathways. All human proteins that are targeted by the virus are part of 592 protein pathways (inset, dotted line). Randomizing human protein sets that are attacked by the virus we find significantly larger numbers of affected pathways, indicating the focused placement of host-pathogen interactions to affect a distinct set of pathways (inset, $P<10^{-3}$ ). (b) A low value of the pathway participation coefficient represents the observation that the interactions of a protein are present in many different pathways and vice versa. Considering all human proteins, we obtain a maximum around low values. Focusing on subsets of targeted human proteins we find that the original signal is significantly reinforced. 
Figure 3: (a) We connect a pair of HIV-1 proteins if both viral proteins target the same set of human proteins. While the size of nodes indicates the number of links the corresponding node is involved in, the thickness of edges refers to the number of co-targeted human proteins, pointing to strong links between Tat, Vpr, Nef and Vif. Assuming that these links mediate biological significance, we elucidate functional sub-networks that emerge from co-regulating human proteins by combinations of HIV proteins. In particular, (b) shows that Tat strongly inhibits subunits of the $26 \mathrm{~S}$ particle of the human proteasome supported by interactions involving Vif. (c) Human proteins that participate in immunological pathways such as interleukines and major histocompatibility complexes are largely affected by a combination of viral proteins, predominantly involving Tat, Nef and Vpr. (d) Tat again plays an important role in stimulating oncogenes that are involved in signal transduction pathways. In particular, we observe that a combination of reverse trancriptase, Vpr and Nef bind p53 while Tat down-regulates this prominent oncogene.

Figure 4: Determining a network of pathways we place a weighted link between pathways if they share proteins that are attacked by the virus. In particular, the size of nodes refers to the number of targeted proteins in the pathway, while the strength of edges indicates the number of such proteins both pathways share, and numbers point to pathways described in SOMTable 2. Considering significantly weighted links $\left(P<10^{-3}\right)$, we observe that the network of attacked pathways is largely composed of four connected components. The densely connected components refer to pathways that play a role in transcription events and mRNA processing, translational and degradation activities. In contrast, the largest component is a surprisingly loose assembly of signaling and regulation pathways, suggesting that the virus needs to interact with a considerable number of such pathways to seize control of the cell. 

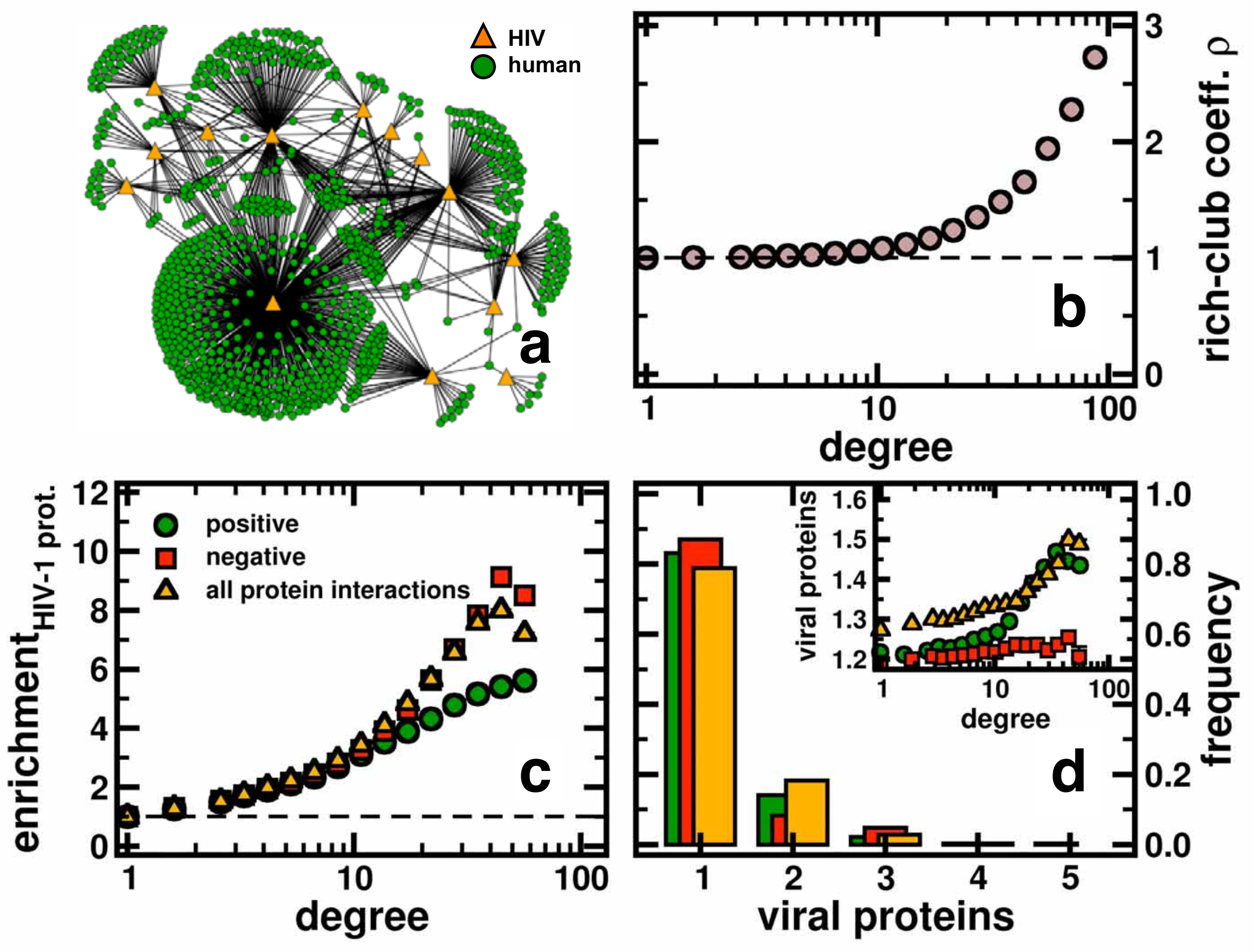

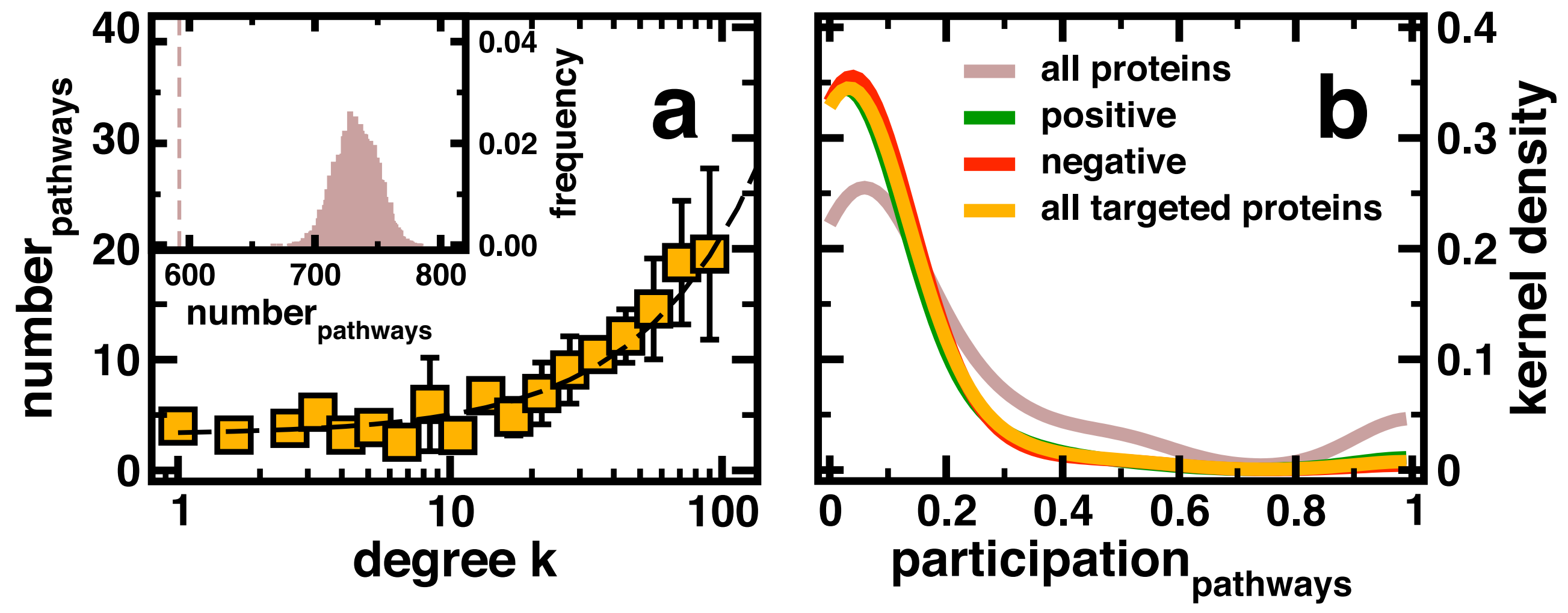

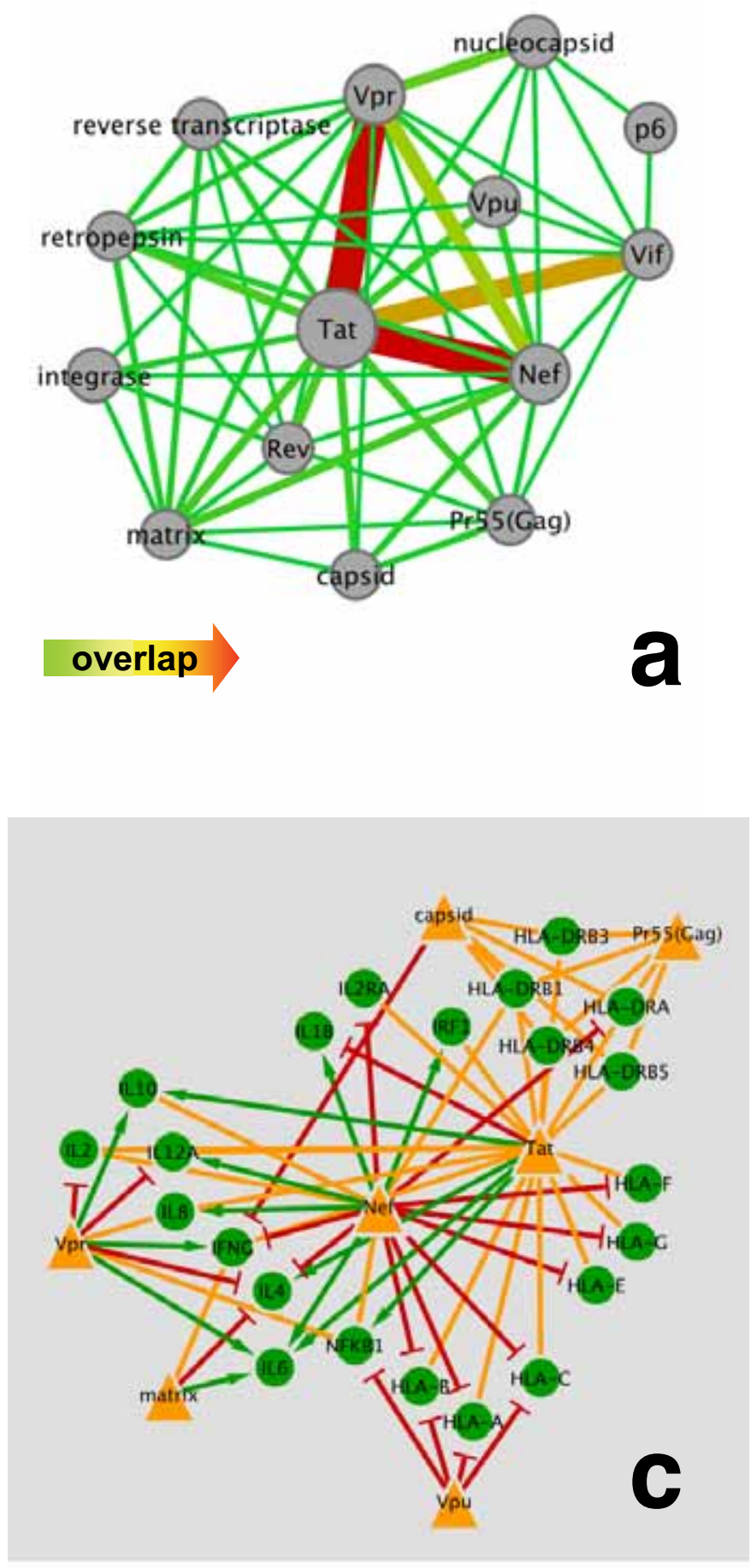
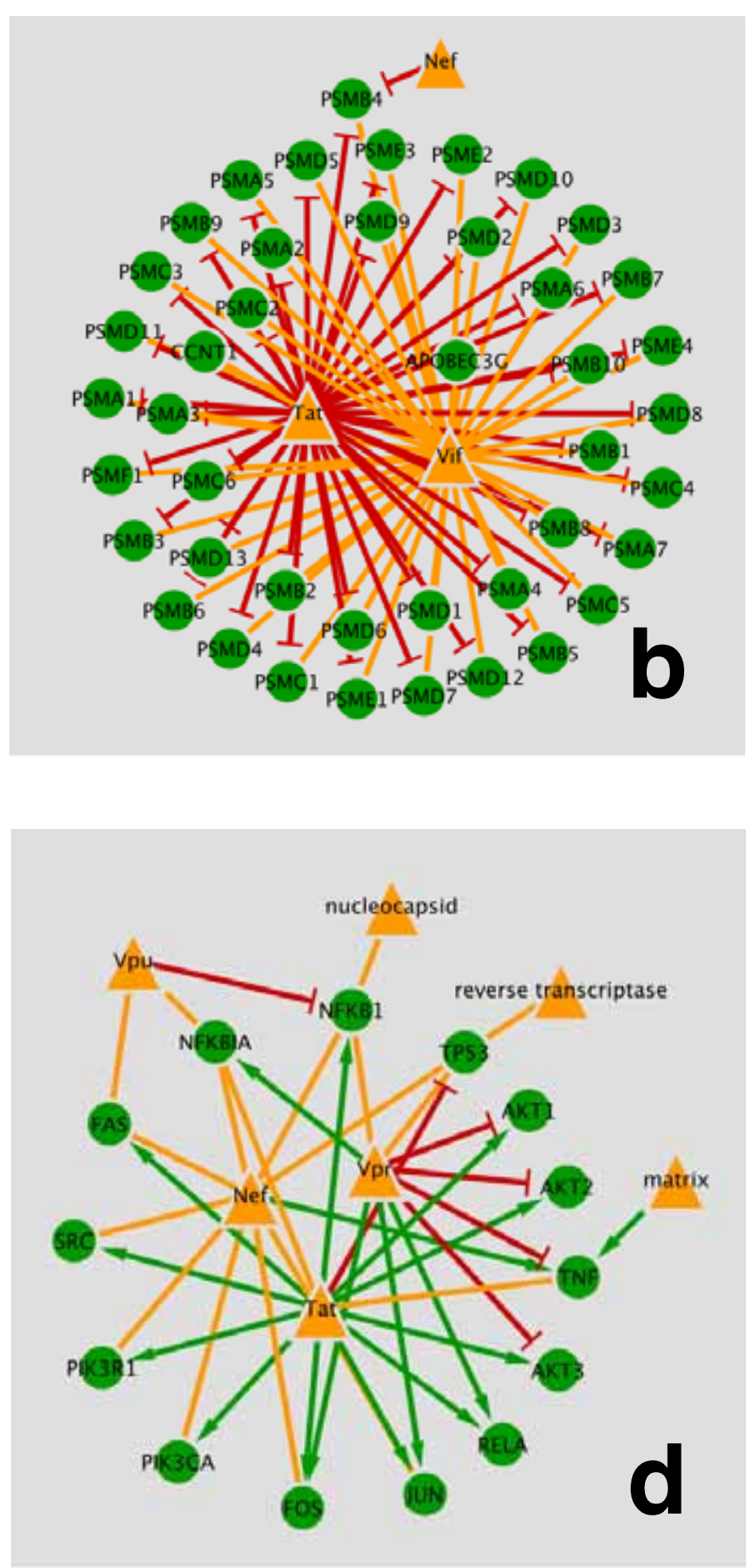


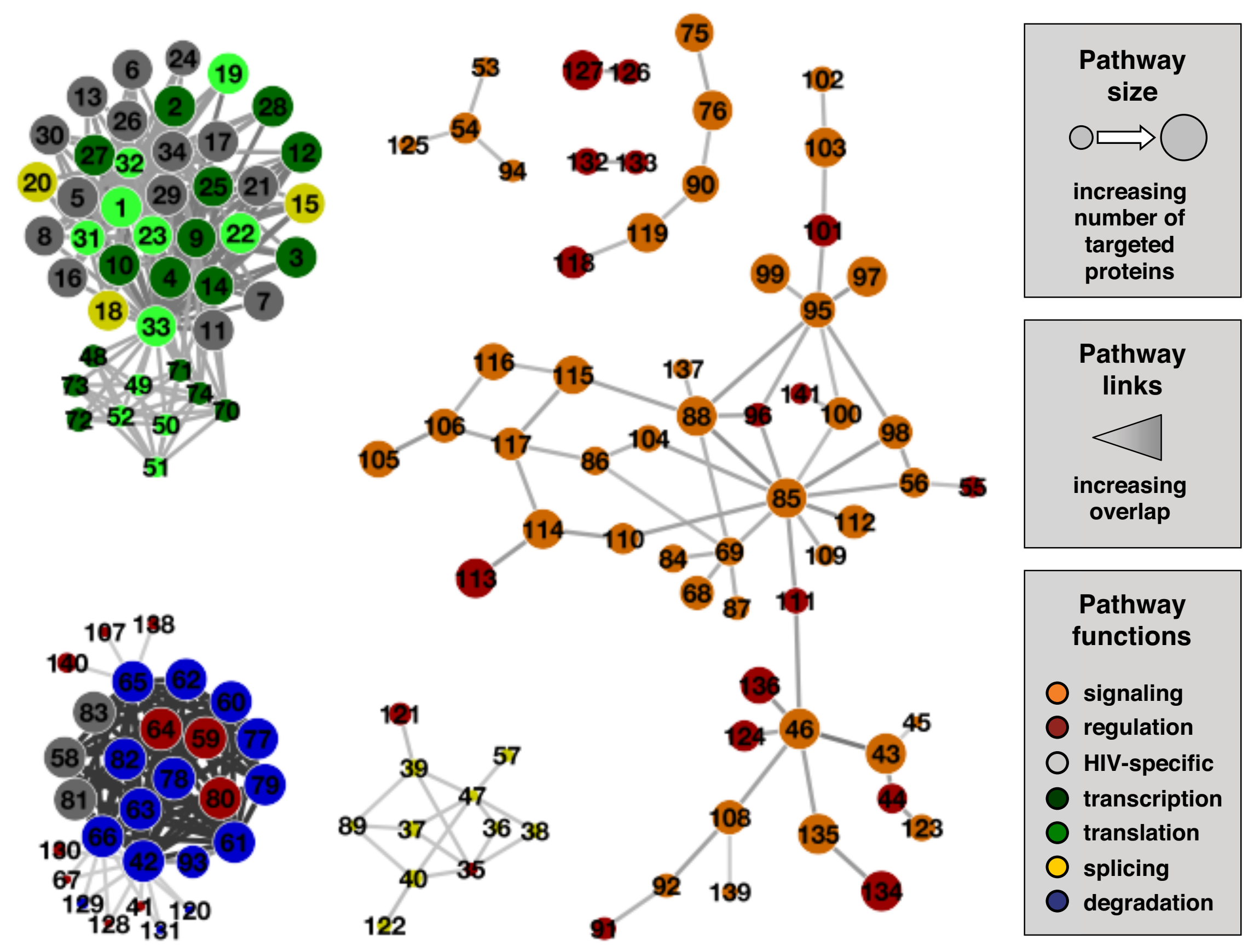

